\title{
Duality in Left-Right Symmetric Seesaw Mechanism
}

\author{
E. Kh. Akhmedov ${ }^{a, b}$ and M. Frigerio ${ }^{c}$ \\ a Physik Department T30, Technische Universität München, \\ James-Franck Straße, D-85748 Garching, Germany \\ ${ }^{b}$ Kurchatov Institute, Moscow, Russia \\ c Department of Physics, University of California, \\ Riverside, CA 92521, USA
}

\begin{abstract}
We consider type I+II seesaw mechanism, where the exchanges of both right-handed neutrinos and isotriplet Higgs bosons contribute to the neutrino mass. Working in the left-right symmetric framework and assuming the mass matrix of light neutrinos $m_{\nu}$ and the Dirac-type Yukawa couplings to be known, we find the triplet Yukawa coupling matrix $f$, which carries the information about the masses and mixing of the right-handed neutrinos. We show that in this case there exists a duality: for any solution $f$, there is a dual solution $\hat{f}=m_{\nu} / v_{L}-f$, where $v_{L}$ is the VEV of the triplet Higgs. Thus, unlike in pure type I (II) seesaw, there is no unique allowed structure for the matrix $f$. For $n$ lepton generations the number of solutions is $2^{n}$. We develop an exact analytic method of solving the seesaw non-linear matrix equation for $f$.

PACS numbers: 14.60.Pq, 14.60.St, 11.30.Er
\end{abstract}

1. Introduction. The discovery of neutrino oscillations in atmospheric, solar, reactor and long-baseline neutrino experiments [1] gave an unambiguous evidence for neutrino mass and mixing and spurred the activity in neutrino mass model building. Among the possible mechanisms of neutrino mass generation, arguably the simplest and most attractive one is the seesaw mechanism [2, 3], which explains the smallness of the neutrino masses through the exchange of superheavy particles. In addition, it has an elegant built-in mechanism of generation of the baryon asymmetry of the universe - baryogenesis via leptogenesis [4].

In its simplest version, the seesaw mechanism extends the standard electroweak model by assuming the existence of $S U(2)_{L}$-singlet right-handed (RH) neutrinos $N_{R}$ with a bare Majorana mass matrix $M_{R}$. The $\mathrm{RH}$ neutrino masses, not being protected by the electroweak symmetry, are naturally large - at Planck or GUT scale. The masses of light neutrinos, produced in this (so-called type I) seesaw mechanism, are 2]

$$
m_{\nu} \simeq m_{\nu}^{I}=-m_{D} M_{R}^{-1} m_{D}^{T},
$$

where $m_{D}=v y, v \simeq 174 \mathrm{GeV}$ being the electroweak VEV and $y$ the Dirac-type Yukawa matrix describing the coupling of lepton doublets to $N_{R}$ and the standard Higgs doublet. Alternatively, the masses of light neutrino can be generated through their interaction with a heavy $S U(2)_{L}$-triplet Higgs scalar $\Delta_{L}$, which can acquire an induced VEV $v_{L}$ through its coupling to the Higgs doublet. This (so-called type II) seesaw mechanism [3] yields

$$
m_{\nu}=m_{\nu}^{I I}=v_{L} f_{L} \sim\left(v^{2} / M_{\Delta}\right) f_{L},
$$

where $f_{L}$ is the Yukawa coupling between the lepton doublets and $\Delta_{L}$, and $M_{\Delta}$ is the mass of the isotriplet Higgs.

The RH neutrinos $N_{R}$, being electroweak singlets, are actually aliens to the standard electroweak model, though can be freely added to it. They are much more natural in models with left-right (LR) symmetry [5] or in $S O(10)$ GUT [6], which can be broken down to the standard model through the LR-symmetric stage. The LR symmetry provides a very natural explanation of the observed maximal parity and $\mathrm{C}$ violation in low-energy weak interactions and is therefore likely to be present at some level in the final theory.

In this class of models, the $\mathrm{RH}$ neutrinos are in doublets $l_{R}$ of $S U(2)_{R}$, and their mass is generated by the VEV $v_{R}$ of the $S U(2)_{R}$-triplet Higgs field $\Delta_{R}$ through the Majorana-like Yukawa coupling. LR symmetry then implies that there should also exist an $S U(2)_{L}$-triplet Higgs field $\Delta_{L}$, and the Yukawa couplings of the triplet Higgs scalars to leptons have the form

$$
f_{L}\left(l_{L}^{T} C i \tau_{2} \Delta_{L} l_{L}\right)+f_{R}\left(l_{L}^{c T} C i \tau_{2} \Delta_{R} l_{L}^{c}\right)+\text { h.c. },
$$

where $l_{L}^{c} \equiv C{\overline{l_{R}}}^{T}, \Delta_{L, R}=\tau \Delta_{L, R}$ and $C$ is the charge conjugation matrix. The Dirac-type Yukawa matrix $y$ comes from the coupling of the lepton doublets $l_{L}$ and $l_{R}$ with the bi-doublet Higgs field $\Phi$. In this class of models, light neutrino masses naturally get both type I and type II contributions, with $M_{R} \equiv v_{R} f_{R}$.

In addition to the gauge LR symmetry, the models of this kind are usually assumed to possess a discrete LR symmetry, which is broken at a scale $v_{L R}$ that may or may not coincide with the $S U(2)_{R}$ breaking scale $v_{R}$. There are essentially two ways in which the discrete LR symmetry can be introduced. In the first one, the lefthanded and $\mathrm{RH}$ fermions are interchanged, while the Higgs fields undergo $\Delta_{L} \leftrightarrow \Delta_{R}^{*}, \Phi \leftrightarrow \Phi^{\dagger}$. This yields

$$
f_{L}=f_{R}^{*}, \quad y=y^{\dagger},
$$

where the last equality holds when the VEVs of the bi-doublet $\Phi$ are both real. The second realization re- 
quires the invariance w.r.t. $l_{L} \leftrightarrow l_{L}^{c}, \Delta_{L} \leftrightarrow \Delta_{L}^{c} \equiv \Delta_{R}$, $\Phi \leftrightarrow \Phi^{c} \equiv \Phi^{T}$, i.e. is essentially the charge conjugation symmetry. It leads to

$$
f_{L}=f_{R} \equiv f, \quad y=y^{T} .
$$

While both implementations of the discrete LR symmetry are possible, the second one is more natural in the context of $S O(10)$ unified theories, where it is an automatic gauge symmetry 7 . In what follows we will adhere to this latter realization, i.e. will be assuming the equalities in eq. (5) to hold. The light neutrino mass matrix in this LR-symmetric seesaw mechanism is then given by

$$
m_{\nu} \simeq m_{\nu}^{I I}+m_{\nu}^{I}=v_{L} f-v^{2} y\left(v_{R} f\right)^{-1} y .
$$

For the realization (4) of discrete LR symmetry, type I term in eq. (66) would contain $\left(f^{*}\right)^{-1}$ rather than $f^{-1}$.

2. Seesaw and duality. We will pursue a bottomup approach: assuming $m_{\nu}$ and $y$ to be known, we solve eq. (6) for the $n \times n$ matrix $f$, where $n$ is the number of lepton generations. Note that the matrices $m_{\nu}$ and $f$ are in general complex symmetric, whereas $y$ is symmetric due to the assumed discrete LR symmetry (5).

An examination of eq. (6) reveals the existence of its following duality property: if $f$ is a solution, so is

$$
\hat{f} \equiv \frac{m_{\nu}}{v_{L}}-f,
$$

provided that the matrix $y$ is invertible. This can be readily verified by the direct substitution of (7) into eq. (6). Notice that exactly the same duality holds in the case of the realization (4) of the discrete left-right symmetry.

The duality implies that, given $m_{\nu}$ and $y$, there is no unique solution for the triplet Yukawa coupling $f$. As we shall see, for $n$ fermion generations there are $2^{n}$ matrices $f$ that, for a given $y$, result in exactly the same mass matrix of light neutrinos $m_{\nu}$. It should be stressed that this duality is a unique property of the LR-symmetric type I+II seesaw: no such duality exists in the cases of pure type I or II seesaw, nor in the case when the two seesaw contributions are unrelated.

In LR symmetric models with non-minimal particle content, the presence of extra particles may complicate the structure of the seesaw. In that case, in general there is no duality (at least in the form discussed in this paper).

We shall now consider the illustrative examples of one and two lepton generations as well as the realistic three generations case.

3. One lepton generation. In this case $m_{\nu}, y$ and $f$ are merely complex numbers. Eq. (6) is a quadratic equation for $f$ with solutions

$$
f_{ \pm}=\frac{m_{\nu}}{2 v_{L}} \pm \sqrt{\frac{m_{\nu}^{2}}{4 v_{L}^{2}}+\frac{v^{2} y^{2}}{v_{L} v_{R}}} .
$$

The quantities $f_{ \pm}$satisfy $f_{-}+f_{+}=m_{\nu} / v_{L}$, i.e. are duals of each other. In the limit $\left|m_{\nu}\right|^{2} \gg 4\left|y^{2} v^{2} v_{L} / v_{R}\right|$, eq. (8) yields

$$
f_{-} \simeq-\frac{y^{2} v^{2}}{m_{\nu} v_{R}}, \quad f_{+} \simeq \frac{m_{\nu}}{v_{L}}+\frac{y^{2} v^{2}}{m_{\nu} v_{R}} .
$$

This limit leads to the dominance of type I or II seesaw in the light neutrino mass, depending on which solution, $f_{-}$or $f_{+}$, is realized in nature. For $v_{L} \ll\left|m_{\nu}\right|$, the solution $f_{+}$violates the perturbative unitarity and must be discarded, so that only $f_{-}$can be acceptable; a necessary condition for either solution to be physical is $\left|v_{R}\right| \gtrsim\left|v^{2} y^{2} / m_{\nu}\right|$.

When the two terms under the square root in eq. (8) are of the same order, the contributions of type I and type II terms to $m_{\nu}$ are also of the same order (mixed seesaw). Finally, in the limit $\left|m_{\nu}\right|^{2} \ll 4\left|y^{2} v^{2} v_{L} / v_{R}\right|$, one has $m_{\nu}^{I} \simeq-m_{\nu}^{I I}$ and $\left|m_{\nu}^{I, I I}\right| \gg\left|m_{\nu}\right|$, i.e. in this case there is a strong cancellation of the two terms in eq. (6).

Consider now $n$ fermion generations with no mixing (which means that $f$ and $y$ are diagonal in the same basis). This case can be described as $n$ replicas of the one-generation case: there exist $n$ pairs of solutions $f_{ \pm}^{(i)}$ $(i=1, \ldots, n)$ to $n$ quadratic equations, and each solution for the matrix $f$ corresponds to picking one value $f_{+}^{(i)}$ or $f_{-}^{(i)}$ from each of $n$ pairs. The number of solutions is thus $2^{n}$. Switching on the mixing will change the nature of the solutions (which will no longer be diagonal), but not their number $[8]$.

4. Two lepton generations. In this case $m_{\nu}, y$ and $f$ are symmetric $2 \times 2$ complex matrices. For definiteness, we shall consider the $\left(\nu_{\mu}, \nu_{\tau}\right)$ sector of $m_{\nu}$. The matrices $f^{-1}$ and $y$ are given by

$$
f^{-1}=\frac{1}{F}\left(\begin{array}{cc}
f_{33} & -f_{23} \\
-f_{23} & f_{22}
\end{array}\right), \quad y=\left(\begin{array}{ll}
y_{\mu 2} & y_{\mu 3} \\
y_{\tau 2} & y_{\tau 3}
\end{array}\right),
$$

where $F \equiv \operatorname{det} f$ and $y_{\tau 2}=y_{\mu 3}$. Defining $x \equiv v_{L} v_{R} / v^{2}$, one can rewrite eq. (6) in components as

$$
\begin{aligned}
x F\left(f_{22}-m_{\mu \mu}\right)= & f_{33} y_{\mu 2}^{2}-2 f_{23} y_{\mu 2} y_{\mu 3}+f_{22} y_{\mu 3}^{2}, \\
x F\left(f_{33}-m_{\tau \tau}\right)= & f_{33} y_{\mu 3}^{2}-2 f_{23} y_{\mu 3} y_{\tau 3}+f_{22} y_{\tau 3}^{2}, \\
x F\left(f_{23}-m_{\mu \tau}\right)= & f_{33} y_{\mu 2} y_{\mu 3}-f_{23}\left(y_{\mu 2} y_{\tau 3}+y_{\mu 3}^{2}\right) \\
& +f_{22} y_{\mu 3} y_{\tau 3},
\end{aligned}
$$

where $m \equiv m_{\nu} / v_{L}$. This is a system of coupled cubic equations for the matrix elements of $f$. Usually, solving systems of this kind presents serious difficulties; one has to resort to numerical methods, whose application is complicated by the fact that non-linear equations have multiple solutions, and their number is often not known in advance. We shall, however, show now that the system of equations (11) admits a simple exact analytic solution. To find this solution, we develop here a method that may also prove to be useful for solving similar systems of coupled non-linear equations appearing in different contexts.

Let us rescale all the matrices of interest according to

$$
f_{i j}=\sqrt{\lambda} f_{i j}^{\prime}, \quad m_{i j}=\sqrt{\lambda} m_{i j}^{\prime}, \quad y_{i j}=\sqrt{\lambda} y_{i j}^{\prime},
$$


where $\lambda$ is an as yet arbitrary complex number. The scaling law was chosen in such a way that in terms of the primed variables the system of equations for $f_{i j}^{\prime}$ has the same form as eq. (11). Next, we fix the value of $\lambda$ by requiring $F^{\prime} \equiv \operatorname{det} f^{\prime}=1$. The system of equations for $f_{i j}^{\prime}$ then becomes linear and can be readily solved. Expressing the primed variables back through the unprimed ones and substituting them into the condition $F^{\prime}=1$, one obtains a 4 th order characteristic equation for $\lambda$. Solving it completes the solution of the problem.

Since the matrix $y$ is symmetric, one can go, without a loss of generality, to the basis where $y$ is diagonal: $y=$ $\operatorname{diag}\left(y_{2}, y_{3}\right)$. The solution for the matrix $f$ then takes the form

$$
f=\frac{x \lambda}{(x \lambda)^{2}-y_{2}^{2} y_{3}^{2}}\left(\begin{array}{cc}
x \lambda m_{\mu \mu}+y_{2}^{2} m_{\tau \tau} & m_{\mu \tau}\left(x \lambda-y_{2} y_{3}\right) \\
\ldots & x \lambda m_{\tau \tau}+y_{3}^{2} m_{\mu \mu}
\end{array}\right),
$$

where $\lambda$ has to be found from the characteristic equation

$$
\begin{array}{r}
{\left[(x \lambda)^{2}-y_{2}^{2} y_{3}^{2}\right]^{2}-x\left[\operatorname{det} m\left(x \lambda-y_{2} y_{3}\right)^{2} x \lambda\right.} \\
\left.+\left(m_{\mu \mu} y_{3}+m_{\tau \tau} y_{2}\right)^{2}(x \lambda)^{2}\right]=0 .
\end{array}
$$

Eq. (14) is quartic in $\lambda$ and so has in general four complex solutions, leading to four solutions for the matrix $f$, as expected.

Rewriting eq. (6) as $x \hat{f}=-y f^{-1} y$ and taking the determinants of both sides, one finds $x^{2} F \hat{F}=y_{2}^{2} y_{3}^{2}$, where $\hat{F} \equiv \operatorname{det} \hat{f}$. Since the condition $F^{\prime}=1$ corresponds to $F=\lambda$, this equation leads to $x^{2} \lambda \hat{\lambda}=y_{2}^{2} y_{3}^{2}$. With the help of this relation, it is straightforward to check that the four structures of $f$ defined by eqs. (13) and (14) form two dual pairs, and it is also easy to express the four solutions of eq. (14) in a closed form:

$x \lambda_{i}=\frac{1}{4}\left[x \operatorname{det} m+r_{ \pm} \pm \sqrt{2(\operatorname{det} m)^{2} x^{2}+4 k x+2 x r_{ \pm} \operatorname{det} m}\right]$,

where

$$
\begin{aligned}
r_{ \pm} & = \pm \sqrt{(\operatorname{det} m)^{2} x^{2}+4 k x+16 y_{2}^{2} y_{3}^{2}}, \\
k & =m_{\mu \mu}^{2} y_{3}^{2}+2 m_{\mu \tau}^{2} y_{2} y_{3}+m_{\tau \tau}^{2} y_{2}^{2} .
\end{aligned}
$$

Eq. (15) gives two pairs of dual solutions, one of them corresponding to $r_{+}$and another to $r_{-}$. In each dual pair the solutions differ by the sign in front of the radical.

From eq. (13) one can see that when a solution $\lambda_{1}$ satisfies $\left|x \lambda_{1}\right| \gg\left|y_{i} y_{j}\right|(i, j=2,3)$, one obtains $f_{1} \simeq m$, which corresponds to type II seesaw case. Then the dual solution $x \lambda_{2}=x \hat{\lambda}_{1}=y_{2}^{2} y_{3}^{2} /\left(x \lambda_{1}\right)$ has modulus $\ll\left|y_{i} y_{j}\right|$ and the corresponding matrix $f_{2}=\hat{f}_{1}$ takes the form obtained in type I seesaw case. Notice that eqs. (15) and (16) imply that the condition $\left|x \lambda_{1}\right| \gg\left|y_{i} y_{j}\right|$ can only be satisfied in the limit $\left|m_{\alpha \beta} m_{\gamma \delta}\right| \gg 4\left|y_{i} y_{j} / x\right|(\alpha, \beta, \gamma, \delta=$ $\mu, \tau)$. This condition ensures the existence of solutions with the dominance of one seesaw type, in analogy with the one generation case. However, in general not all four solutions correspond to the one seesaw type dominance in this limit: if $|\operatorname{det} m| \gg 4\left|y_{i} y_{j} / x\right|$, the remaining two dual solutions $f_{3}$ and $f_{4}$ are of mixed type. When $\left|m_{\alpha \beta} m_{\gamma \delta}\right| \lesssim$ $4\left|y_{i} y_{j} / x\right|$, in general all four solutions are of mixed type. A similar classification applies to the 3 -generation case.

5. Three lepton generations. In this case $f, y$ and $m=m_{\nu} / v_{L}$ are complex symmetric $3 \times 3$ matrices. As in the 2-generation case, we go to the basis where $y$ is diagonal: $y=\operatorname{diag}\left(y_{1}, y_{2}, y_{3}\right)$. Quite analogously to the derivation of eq. (11), one can obtain from (6) equations for the matrix elements of $f$ and its dual $\hat{f}$ :

$$
\begin{aligned}
& x F\left(f_{i j}-m_{i j}\right)=y_{i} y_{j} F_{i j}, \\
& x \hat{F}\left(\hat{f}_{i j}-m_{i j}\right)=-x \hat{F} f_{i j}=y_{i} y_{j} \hat{F}_{i j},
\end{aligned}
$$

where $F \equiv \operatorname{det} f, \hat{F} \equiv \operatorname{det} \hat{f}$ as before, and

$$
\begin{aligned}
& F_{i j} \equiv \frac{1}{2} \epsilon_{i k l} \epsilon_{j m n} f_{k m} f_{l n}, \\
& \hat{F}_{i j} \equiv \frac{1}{2} \epsilon_{i k l} \epsilon_{j m n} \hat{f}_{k m} \hat{f}_{l n}=M_{i j}-T_{i j}+F_{i j}, \\
& M_{i j} \equiv \frac{1}{2} \epsilon_{i k l} \epsilon_{j m n} m_{k m} m_{l n}, \quad T_{i j} \equiv \epsilon_{i k l} \epsilon_{j m n} f_{k m} m_{l n} .
\end{aligned}
$$

Taking the determinants of both sides of the equation $x \hat{f}=-y f^{-1} y$, one obtains

$$
x^{3} F \hat{F}=-y_{1}^{2} y_{2}^{2} y_{3}^{2} .
$$

Eq. (17) is a system of six coupled quartic equations for the elements $f_{i j}$ (note that $F$ is cubic in $f_{i j}$ in the 3 -generation case). Since the right-hand sides of these equations are quadratic rather than linear in $f_{i j}$, a simple rescaling would not immediately linearize the system. However, using the dual system of equations (18) one can write for these right-hand sides $y_{i} y_{j} F_{i j}=$ $-x \hat{F} f_{i j}+y_{i} y_{j}\left(T_{i j}-M_{i j}\right)$. The resulting system of equations can now be linearized by a rescaling, similar to that in eq. (12), except that the scaling factor is now $\lambda^{1 / 3}$ rather than $\sqrt{\lambda}$. Once again, we fix $\lambda$ by requiring $F^{\prime}(\lambda)=1$; eq. (19) then yields $\hat{F}^{\prime}=-\left(y_{1}^{\prime} y_{2}^{\prime} y_{3}^{\prime}\right)^{2} / x^{3}$. The linearized system is

$$
\left[x^{3}-\left(y_{1}^{\prime} y_{2}^{\prime} y_{3}^{\prime}\right)^{2}\right] f_{i j}^{\prime}-x^{3} m_{i j}^{\prime}=x^{2} y_{i}^{\prime} y_{j}^{\prime}\left(T_{i j}^{\prime}-M_{i j}^{\prime}\right) .
$$

The characteristic equation for $\lambda$ is now of 8 th order and its solutions lead to four pairs of dual solutions $f$ and $\hat{f}$ for the system (17). This 8th order equation and the resulting general analytic solution for $f$ are rather lengthy and will be given elsewhere; here we present the simplified case $y_{1} \rightarrow 0$.

The case $\left|y_{1}\right| \ll\left|y_{2,3}\right|$ is actually physically well motivated, in view of the smallness of the masses of firstgeneration charged fermions. Notice that for $y_{1}=0$ the matrix $y$ is not invertible, so that the proof of the duality of solutions for $f$ breaks down. However, one can check that the solutions of eq. (17) for $y_{1}=0$ are the same as the ones obtained by setting $y_{1}=0$ in eq. (20). In other words, one can safely use the duality and take the limit $y_{1} \rightarrow 0$ in the final result. The solution for the symmetric matrix $f$ is then given in components as

$$
\begin{gathered}
f_{11}=m_{e e}, \quad f_{12}=m_{e \mu}, \quad f_{13}=m_{e \tau}, \\
f_{23}=\left(m_{\mu \tau}+\frac{y_{2} y_{3} m_{e \mu} m_{e \tau}}{x \lambda}\right) / d_{2},
\end{gathered}
$$




$$
\begin{gathered}
f_{22}=\left[m_{\mu \mu}+\frac{y_{2}^{2}}{x \lambda}\left(M_{22}-\frac{y_{3}^{2} m_{e e} m_{e \mu}^{2}}{x \lambda}\right)\right] / d_{1}, \\
f_{33}=\left[m_{\tau \tau}+\frac{y_{3}^{2}}{x \lambda}\left(M_{33}-\frac{y_{2}^{2} m_{e e} m_{e \tau}^{2}}{x \lambda}\right)\right] / d_{1},
\end{gathered}
$$

where

$$
d_{1}=1-\frac{y_{2}^{2} y_{3}^{2} m_{e e}^{2}}{(x \lambda)^{2}}, \quad d_{2}=1+\frac{y_{2} y_{3} m_{e e}}{x \lambda} .
$$

In the considered limit $y_{1} \rightarrow 0$ the characteristic equation for $\lambda$ reduces to

$$
\begin{array}{r}
\lambda^{4}\left\{\left[(x \lambda)^{2}-m_{e e}^{2} y_{2}^{2} y_{3}^{2}\right]^{2}-x\left[\operatorname{det} m\left(x \lambda-m_{e e} y_{2} y_{3}\right)^{2} x \lambda\right.\right. \\
\left.\left.+\left(M_{22} y_{2}+M_{33} y_{3}\right)^{2}(x \lambda)^{2}\right]\right\}=0 .
\end{array}
$$

A comment on the duality and multiplicity of solutions in the 3 -generation case is in order. If a solution $\lambda$ of the general characteristic equation is non-zero in the limit $y_{1} \rightarrow 0$, then $\hat{\lambda}=-y_{1}^{2} y_{2}^{2} y_{3}^{2} /\left(x^{3} \lambda\right) \rightarrow 0$, which means that the determinant of $\hat{f}$ vanishes. Thus, the dual of any solution that is finite for $y_{1} \rightarrow 0$ becomes singular and must be discarded. Therefore, in this limit there are only four (rather than eight) solutions with no duals. The corresponding values of $\lambda$ are the zeros of the factor in curly brackets in eq. (23), which is quartic in $\lambda$. From the comparison with eq. (14) a strong connection with the pure 2 -generation case is evident, so that a different duality among the four remaining solutions exists: if $\lambda$ satisfies eq. (23), so does $\tilde{\lambda} \equiv y_{2}^{2} y_{3}^{2} m_{e e}^{2} /\left(x^{2} \lambda\right)$, and it corresponds to $\tilde{f} \equiv \tilde{m}-f$, where $\tilde{m}_{\alpha \beta}=m_{\alpha \beta}+m_{e \alpha} m_{e \beta} / m_{e e}$. There are two pairs of such solutions.

6. Concluding remarks. We have revealed and analyzed an interesting duality property of the seesaw mechanism with contributions of both RH neutrinos and isotriplet Higgs bosons in the presence of discrete leftright symmetry. In particular, it has been shown that for a given mass matrix of light neutrinos $m_{\nu}$ and Diractype Yukawa coupling matrix $y$, there are multiple solutions for the Majorana-type Yukawa coupling matrix $f$ and thus for the matrix of RH neutrinos $M_{R}$. The number of solutions, however, does not exceed eight (for three lepton generations), and with the help of the developed here formalism they can be readily analyzed one by one. In contrast to this, in models where type I and type II contributions to neutrino mass are unrelated, there are in general infinitely many possible decompositions of $m_{\nu}$ into these two parts.

The discrete LR symmetry of the underlying theory must be broken at some scale $v_{L R}$, and the renormalization group evolution below this scale may result in a violation of conditions (5) at lower energies. The corrections to the matrix elements of $y$ and $f$ depend logarithmically on the ratios of the masses of $\mathrm{RH}$ neutrinos and Higgs triplets and are suppressed by loop factors and possibly by small couplings. We checked numerically that the reconstruction of the matrix $f$ as performed in this paper remains accurate at a percent level if the LR-violating corrections to the matrix elements are of the order of percent. This stability, however, may be lost if small matrix elements receive corrections proportional to the large ones. This issue requires a dedicated study, which is beyond the scope of the present paper.

Possible applications of our results include the bottomup reconstruction of the mass matrix of heavy $\mathrm{RH}$ neutrinos and model building. The bottom-up approach requires the knowledge of the light neutrino mass matrix and of the Dirac-type Yukawa couplings $y$. The former is partially known from the experiment (absolute mass scale, 1 - 3 mixing angle and $\mathrm{CP}$ violating phases have not been measured yet), whereas for $y$ one needs both data and additional theoretical assumptions, such as quarklepton symmetry or Grand Unification. Model builders can use our results to immediately find out the allowed structures of the triplet Yukawa couplings $f$ that can successfully reproduce the low energy phenomenology, which may help them to systematically look for the underlying symmetries of the mechanism of lepton mass generation.

We thank R. N. Mohapatra, V. A. Rubakov, G. Senjanović and especially A. Yu. Smirnov for useful discussions. This work was supported by SFB-375 für Astro-Teilchenphysik der Deutschen Forschungsgemeinschaft (EA) and by U.S. Department of Energy grant no. DE-FG03-94ER40837 (MF).
[1] See, e.g., G. L. Fogli et al., hep-ph/0506083

[2] P. Minkowski, Phys. Lett. B 67, 421 (1977); M. GellMann, P. Ramond and R. Slansky, In Supergravity, P. van Nieuwenhuizen and D.Z. Freedman (eds.), North Holland Publ. Co., 1979, p. 315; T. Yanagida, In Proc. Workshop on the Baryon Number of the Universe and Unified Theories, O. Sawada and A. Sugamoto (eds.), Tsukuba, Japan, 13-14 Feb. 1979, p. 95; S. L. Glashow, In Proc. Cargese 1979 Quarks and Leptons, p. 687; R. N. Mohapatra and G. Senjanović, Phys. Rev. Lett. 44, 912 (1980).

[3] M. Magg and C. Wetterich, Phys. Lett. B 94, 61 (1980); G. Lazarides, Q. Shafi and C. Wetterich, Nucl. Phys. B 181, 287 (1981); J. Schechter and J. W. F. Valle, Phys.
Rev. D 22, 2227 (1980); R. N. Mohapatra and G. Senjanović, Phys. Rev. D 23, 165 (1981).

[4] M. Fukugita and T. Yanagida, Phys. Lett. B 174, 45 (1986).

[5] J. C. Pati and A. Salam, Phys. Rev. D 10, 275 (1974); R. N. Mohapatra and J. C. Pati, Phys. Rev. D 11, 566 (1975); G. Senjanović and R. N. Mohapatra, Phys. Rev. D 12, 1502 (1975).

[6] H. Georgi, AIP Conf. Proc. 23 (1975) 575; H. Fritzsch and P. Minkowski, Annals Phys. 93, 193 (1975).

[7] E.g., C. S. Aulakh et al., Phys. Rev. D 58, 115007 (1998).

[8] We thank A. Yu. Smirnov for this argument. 